\title{
Selection of the size of field of view in thermal imaging observations of small areas
}

\author{
Krzysztof Dziarki*, Arkadiusz Hulewicz, and Zbigniew Krawiecki ${ }^{1}$ \\ ${ }^{1}$ Institute of Metrology and Optoelectronics, Poznan University of Technology, ul. Piotrowo 3A 60-965 Poznań, Poland
}

\begin{abstract}
The paper explains the effect of matrix resolution on the field of view of a single detector. The dependency of the field of view of a single detector as a function of distance between the lens of thermal imaging camera and the surface observed is shown. It was proposed how to select the lens of thermal imaging camera so that the obtained thermogram it would be able to achieve such a geometric resolution that would allow for sufficiently accurate mapping of temperature distribution on the surface observed.
\end{abstract}

\section{Preface}

In the course of thermovision measurements, it is necessary from time to time to determine the temperature of a small part of surface. This happens, for example, while observing the casings of electronic components. Due to the dimensions of casings, the surfaces considered are small (of the order of square micrometres). Performing a measurement with a smaller error causes that the final measurement error of the entire measurement system is smaller $[1,2]$. The question is justified: will your thermal imaging camera allow obtaining a thermogram with a geometric resolution to sufficiently accurately determine the reliable temperature of the surface observed?

\section{Field of view of a single detector}

In order to obtain reliable information on the temperature of the surface observed, it must be at least three times bigger than the field of view of a single IR detector of the thermal imaging camera used. The optical properties of the IR detector matrix resemble the optical dependencies of the CCD matrix [3]. The field of view of a single IR detector depends on the distance between the lens and the observed surface, and the angle of view of the detector. The catalogue parameter describing the viewing angle of a single detector is IFOV (Instantaneous Field of View). This is the angle in the area of which the segment of surface observed has emitted the radiation reaching a single detector. Information on the IFOV value is usually included in the technical documentation of the thermal imaging camera as a single value of an angle expressed in milliradians. It happens that the IFOV catalogue parameter informs about the length of the vertical instantaneous field of view of a single detector - VIFOV, and the horizontal instantaneous field of view of a single detector - HIFOV while maintaining a certain work distance $(W D)$. In order to differentiate the value given in length units, they will be recorded in the further part of this paper with the $\mathrm{m}$ lower index. The IFOV value can also be determined on the basis of the FOV (Field of View), the value of which can be found in the camera's catalogue data sheet. The FOV value is the angle within the area of which the IR radiation was emitted by the field of view of the entire matrix [4]. One can distinguish the $F O V$ value in the vertical plane - VFOV (Vertical Field of View) and in the horizontal plane - HFOV (Horizontal Field of View). While determining the IFOV value on the basis of the $F O V$ value, the values of angles should be converted to radians and divided by the number of detectors in the matrix row or column so that the obtained HIFOV and VIFOV values were approximately equal. For example, for a thermal imaging camera fitted with a lens with a FOV value of $25^{\circ} \times 19^{\circ}$ and a matrix with a resolution of $240 \times 180$ pixels (detectors), the IFOV value can be determined on the basis of equation 1 :

$$
I F O V=19 \cdot 2 \pi / 360 \cdot 180=25 \cdot 2 \pi / 360 \cdot 240=1,8 \mathrm{mrad}
$$

The VIFOV and HIFOV values are approximately equal. Knowing the values of both parameters by using the (2) (4) formulas one may calculate the field of view of a single detector as a function of distance separating the IR detectors matrix of the thermal imaging camera from the surface observed.

$$
\begin{gathered}
V=2 \cdot l \cdot \operatorname{tg}(\text { VIFOV } / 2) \\
H=2 \cdot l \cdot \operatorname{tg}(H I F O V / 2) \\
P=V \cdot H=4 \cdot l^{2} \cdot \operatorname{tg}(V I F O V / 2) \cdot \operatorname{tg}(V I F O V / 2)
\end{gathered}
$$

$P$ - area of the field of view, $V$ - length of the vertical instantaneous field of view, $H$ - length of the horizontal instantaneous field of view, $l$ - distance between the lens and the surface observed.

\footnotetext{
*Corresponding author: Krzysztof.Dziarski@put.poznan.pl
} 
When selecting the field of view of a single detector, one should remember that for a distance shorter than the work distance (value of which can be found in the technical documentation), the thermogram obtained will be out of focus, regardless of the camera settings.

\section{Calculating the field of view of a single IR detector of a camera with additional lens}

Modern thermal imaging cameras designed for scientific and diagnostic purposes have the option of replacing the mounted lens or mounting the additional one. In the case of additional lens, the FOV values are given in angles or units of length (usually in $\mathrm{mm}$ ) as the length of the vertical or horizontal instantaneous field of view obtained while maintaining a specified WD (Work Distance) between the lens and the observed surface. The value of WD is included in the technical documentation of lens. When the $F O V$ values of lens are given in angles, the IFOV values can be obtained as described in point 2. In order to facilitate the operation and selection, manufacturers of additional lens provide information on the IFOV value expressed in units of length, which can be obtained for a given matrix resolution while maintaining $W D$ distance. When the $I F O V m$ value is not included in the documentation in order to obtain it, it is enough to divide the FOVm value by the number of detectors in the line or row of the matrix. For example, using a lens with $F O V$ of $16 \mathrm{~mm} \mathrm{x}$ $12 \mathrm{~mm}$, with a $160 \times 120$ pixel matrix resolution one may obtain the following $I F O V_{m}$ value by using equation (5):

$$
I F O V_{m}=16 / 160=12 / 120=100 \mu \mathrm{m}
$$

By selecting additional lens designed for observation of fragments of the surface of casings of electronic components, one can check how the field of view of a single detector changes over the entire range of distance (between the observed surface and the lens), in which a sharp image can be obtained. To this end, the $\mathrm{FOV}_{m}$ (Field of View) value should be converted to a FOV value expressed in angles. For this purpose, one can use the equations (6) and (7):

$$
\begin{aligned}
& H F O V=2 \cdot \operatorname{arctg}\left(H_{F O V_{m}} / 2 \cdot W D\right) \\
& V F O V=2 \cdot \operatorname{arctg}\left(\operatorname{VFOV}_{m} / 2 \cdot W D\right)
\end{aligned}
$$

Then, based on the value of FOV (HFOV and VFOV), the IFOV value expressed in radians should be calculated. It is possible to do this by using equations (8) and (9):

$$
\begin{aligned}
& H I F O V=H F O V \cdot 2 \pi / 360 \cdot H_{\text {mat }} \\
& V I F O V=V F O V \cdot 2 \pi / 360 \cdot V_{\text {mat }}
\end{aligned}
$$

in which: $H_{\text {mat }}$ - number of detectors in the matrix line, $V_{\text {mat }}$ number of detectors in the matrix column.
The length of the instantaneous field of view expressed in millimetres at a given lens-to-surface distance can be obtained on the basis of the following equations:

$$
\begin{aligned}
& \mathrm{HIFOV}_{m}=2 \cdot l \cdot \operatorname{tg}(\mathrm{HIIFOV} / 2) \\
& \text { IIFOV }_{m}=2 \cdot l \cdot \operatorname{tg}(\mathrm{HIIFOV} / 2)
\end{aligned}
$$

\section{Conclusion}

Making a reliable, thermovision measurement of temperature of a small area requires obtaining a thermogram with adequate sharpness. This is possible, inter alia, thanks to proper selection of the distance between the lens and the surface observed. The size of the field of view of a single detector depends on this distance. When the field of view of a single detector is larger than the surface observed, the surface temperature being measured may differ from the actual temperature. Such a selection of the lens-to-surface distance is not always possible, to ensure at the same time the proper sharpness of thermogram and the value of field of view of a single detector. Reading out the proper parameters of the matrix and the lens of thermal imaging camera and using the indicated dependencies, one can check whether the optical system of your camera allows making a thermogram with the proper geometric resolution while maintaining the sharpness. It is also possible to precisely determine such a lens-to-surface distance to meet both conditions and to determine the value of field of view of a single detector obtained at the setting of this distance. Such a thermal imaging camera can be a part of a measurement system controlled from a computer [5] that transmits measurement results using wireless methods [6].

\section{References}

1. Minkina W., Basics of thermovision measurements. Part III - metrological issues, interpretation of results, PAK 2001.

2. A. Odon, Z. Krawiecki, Meas., 44, 8, 1406-1411, (2011)

3. J. Parzych, Z. Krawiecki, Przeg. Elektrotechniczny, 10, 86-87, (2010)

4. K. Dziarski, J. Parzych, The solutions used for long wave thermographic cameras designed for observing elements in SMD housings, ITM WOC Vol. 19, 2018

5. P. Otomanski, Z. Krawiecki, A. Odon, JPCS, 238, 012005 (2010).

6. A. Cysewska-Sobusiak, A. Hulewicz, M. Boltrukiewicz, D. Prokop, Proc. IEEE VECIMS, 116 (205) 